\title{
Identification of abnormally high expression of POGZ as a new biomarker associated with a poor prognosis in osteosarcoma
}

\author{
Sikuan Zheng, ${ }^{1,2,3,4 *}$ Yue Liu, ${ }^{5 *}$ Haohe Sun, ${ }^{2}$ Jingyu Jia, ${ }^{1,3,4}$ Tianlong Wu, ${ }^{1,3,4}$ Rui Ding, ${ }^{1,2,3,4}$ Xigao Cheng ${ }^{1,3,4}$ \\ ${ }^{1}$ The Second Affiliated Hospital of Nanchang University, Jiangxi \\ ${ }^{2}$ The Second Clinical Medical College of Nanchang University, Jiangxi \\ ${ }^{3}$ Institute of Orthopedics of Jiangxi Province, Nanchang, Jiangxi \\ ${ }^{4}$ Institute of Minimally Invasive Orthopedics of Nanchang University, Jiangxi \\ ${ }^{5}$ Queen Mary School, Medical College of Nanchang University, Nanchang, Jiangxi, China \\ *These authors contributed equally
}

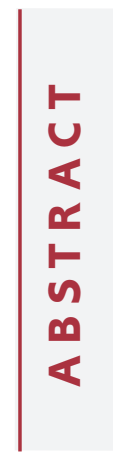

\begin{abstract}
Osteosarcoma (OS) is the most prevalent malignant bone tumor in children and young adults. There is an urgent need for a novel biomarker related to the prognosis of OS. We performed a meta-analysis incorporating six independent datasets and performed a survival analysis with one independent dataset GSE21257 in the GEO database for gene screening. The results revealed that one potential biomarker related to OS survival, POGZ, was the most significantly upregulated gene. We also verified that the $P O G Z$ was overexpressed in clinical samples. The survival analysis revealed that $P O G Z$ is associated with a poor prognosis in OS. Moreover, flow cytometry analysis of isolated OS cells demonstrated that OS cells were arrested in the $\mathrm{G}_{1}$ phase after $P O G Z$ knockdown. The RNA-seq results indicated that $P O G Z$ was co-expressed with $C C N E 1$ and $C C N B 1$. Pathway analysis showed that genes associated with high expression levels of $P O G Z$ were related to the cell cycle pathway. A cell model was constructed to detect the effects of POGZ. After POGZ knockdown, OS cell proliferation, invasion and migration were all decreased. Therefore, $P O G Z$ is an important gene for evaluating the prognosis of OS patients and is a potential therapeutic target.
\end{abstract}

Key words: Prognosis; osteosarcoma; biomarkers; POGZ; cell cycle.

Correspondence: Prof. Xigao Cheng, The Second Affiliated Hospital of Nanchang University, No.1 Minde Road, EastLaker District, Nanchang, Jiangxi, China. Tel.+86.791.8633-1753. E-mail: 228206846@qq.com

Funding: Natural Science Research Program of Jiangxi (No. 20202ACBL206012. to XC); National Natural Science Foundation of China (No. 81660357 to XC); National Natural Science Foundation of China (No. 81860397 to $\mathrm{XC})$.

Contributions: SZ, YL, JJ, XC, conceived and designed the study; SZ, YL, HS, RD, TW, performed the literature search and data extraction; SZ, HS, TW, RD, analyzed the data; SZ, YL, JJ, drafted the manuscript. All the authors have read and approved the final version of the manuscript and agreed to be accountable for all aspects of the work.

Conflict of interest: The authors declare that they have no competing financial interests. 


\section{Introduction}

Osteosarcoma (OS) accounts for approximately $60 \%$ of malignant bone tumors in children and young people, making it the most prevalent tumor of this type; it seriously affects patients' quality of life and results in death due to rapid development of the disease. ${ }^{1,2}$ Treatment options for OS include surgery, chemotherapy and radiotherapy. Despite the fact that advances in treatment methods have increased the five-year survival rate of OS patients to approximately $60-70 \%$, approximately $30 \%-40 \%$ of patients still develop lung metastases or die. ${ }^{3,4}$ It is worth noting that the prognosis of patients with metastatic OS is extremely poor. ${ }^{5}$ Prognostic evaluation and early diagnosis are crucial and can effectively improve the survival of OS patients. ${ }^{6}$ However, currently available biomarkers cannot effectively predict the prognosis of OS patients. ${ }^{7}$ Therefore, there is a pressing clinical need to discover new biomarkers correlated with the prognosis of OS. ${ }^{6-8}$ In medical oncology, highthroughput sequencing and gene chip technology are promising tools used to identify potential molecular targets. They provide new methods to explore OS-related genes and predict new tumor molecular interactions, key regulatory molecules, and therapeutic drug targets. ${ }^{9}$ Recently, these technologies have been successfully utilized to discover new biomarkers in tumors, including the $M Y C^{10,11}$ and $R A S,,^{12}$ which both promote OS metastasis. In addition, $V E G F^{13}$ was found to be positively associated with metastasis and a poor prognosis of in OS patients. Nonetheless, these aforementioned methods do not offer many potential biomarkers related to the prognosis of OS. ${ }^{14}$ Hence, it is of tremendous clinical significance to discover new biomarkers related to the prognosis of OS and explore their molecular mechanisms. To expand the research on molecules related to the prognosis of OS, we identified a gene known as POGZ that is considerably linked to OS survival using bioinformatics. To further evaluate the relationship between the expression level of $P O G Z$ and OS, we constructed a cytological model verifying the effects of $P O G Z$ inhibition on OS cell proliferation and metastasis. $P O G Z$ might be regarded as a potential therapeutic target for OS.

\section{Materials and Methods}

\section{Clinical specimens}

The tissue microarray chip was provided by Typos Biotechnology Company (Xi'an, China) and included 41 OS samples and 19 normal control samples. Table 1 displays the clinicopathological characteristics of the corresponding OS patients.

\section{Data extraction and analysis of screened genes}

In the Gene Expression Omnibus (GEO) database (http://www.ncbi.nlm.nih.gov/geo/), we selected 6 expression profiles and downloaded the original (.CEL file) and platform files. Background correction, quantile normalization, professional summary, $\log 2$ conversion and missing values supplementation of the matrix data for each GEO dataset were performed using the "affy" software package and R/Bioconductor software (version 3.5.3) "limma" package. Subsequently, the "mama" package was used to group OS patients and normal controls, perform a meta-analysis, and obtain the z-score value. We used the "meta" package in the R software to download the pan genome project containing multitumor expression data to draw a forest map.

The Kaplan-Meier survival curve was plotted using the GEO expression profile and the survival data through the $\mathrm{R}$ software "survival" package for single factor Cox survival analysis.

\section{Immunohistochemical staining}

After deparaffinization, the tissue microarray chip was subjected to antigen retrieval and endogenous peroxidase blocking. After serum blocking, polyclonal rabbit anti-human POGZ (1:100, Absin, abs132798) was added and incubated overnight at $4{ }^{\circ} \mathrm{C}$. Next, the secondary antibody (1:100, Absin, abs20044) of the corresponding species was added, and the DAB staining kit was used to (Vector Laboratories, USA) to detect the signal. The histochemical score was calculated using the Quant Center analysis tool: $\mathrm{H}$ score $=\sum(\mathrm{PI} \times \mathrm{I})=($ percentage of weak intensity cells $\times 1)+($ percentage of medium intensity cells $\times 2)+($ percentage of high intensity cells $\times 3){ }^{23}$

\section{Receiver operating characteristic (ROC) curve and logistic regression analysis}

ROC curve analysis was performed to evaluate the sensitivity (true positive rate) and specificity (true negative rate) of $P O G Z$ for OS diagnosis. We also investigated the size of the area under the curve (AUC) by using the "pROC" package of R software.

\section{Biological function and pathway enrichment analysis}

Gene set enrichment analysis (GSEA) is a computational method that determines whether an a priori defined set of genes shows statistically significant, concordant differences between two biological states. It can be used to evaluate microarray data at the gene set level. The Kyoto Encyclopedia of Genes and Genomes (KEGG) is a bioinformatics resource. We can use it to study the relationship between genes. Samples from the GSE42352 datasets were divided into two groups based on the expression levels of POGZ (median value) and the gene set enrichment analysis (GSEA) software (http://software.broadinstitute. org/gsea/index. jsp) was applied for both groups.

\section{Cell culture and transient transfection}

OS cell lines including Saos-2 and U-2OS were supplied by the China Center Type Culture Collection (CCTCC, Shanghai, China). Saos-2 and U-2OS cells were cultured in a DMEM media (Gibco, Waltham, MA) supplemented with 10\% FBS (Gibco, Waltham, MA). All the cells were grown at $37^{\circ} \mathrm{C}$ in $5 \% \mathrm{CO} 2$. Following the manufacturer's recommendations, Turbofect transfection reagent was used (Thermo Fisher Scientific Inc, Shanghai, China) to transfect OS cells with negative control (NC) and POGZ siRNA (GenePharma, Shanghai, China) into OS cells. The siRNA sequences targeting $P O G Z$ were as follows: siPOGZ-1: 5'GCCACGAACUGUUCCUGUATT-3', 5'-UACAGGAACAGUUCGUGGCTT-3'; SiPOGZ-2: 5'-CCUAAUCAUUUCCCUACUUTT-3', 5' -AAGUAGGGAAAUGAUUAGGTT-3'; and siPOGZ-3: 5'-CCACAUGAUCAACAAUCAUTT-3', 5'-

Table 1. Clinicopathological characteristics of OS patients.

\begin{tabular}{lcc} 
& Character & Number of case (\%) \\
Grade & G1 G2 & $17(0.41)$ \\
Sex & G3 G4 & $24(0.59)$ \\
& F & $14(0.34)$ \\
Stage & M & $27(0.66)$ \\
\multirow{2}{*}{ Age } & I $\sim$ II & $38(0.93)$ \\
& III IV & $3(0.07)$ \\
Location & $<20$ & $13(0.32)$ \\
& $>20$ & $28(0.68)$ \\
\hline
\end{tabular}


AUGAUUGUUGAUCAUGUGGTT-3'. The negative control siRNA sequence was as follows: 5'-UUCUCCGAACGUGUCACGUTT-3', 5'-ACGUGACACGUUCGGAGAATT-3'.

\section{RNA extraction and real-time quantitative RT-PCR}

The total amount of RNA from the OS cells was extracted with the AxyPrep Multisource Total RNA Miniprep Kit (Axygen Scientific, Union City, CA, USA) according to the manufacturer's protocol. A cDNA volume of $20 \mu \mathrm{l}$ was synthesized utilizing the Takara PrimeScriptTM RT Reagent Kit and gDNA Eraser (Cat\# RR047A, Lot\# AK2802) using $1 \mu \mathrm{g}$ total RNA following the measurements of total RNA concentration using the software Quantity One software (PDI Inc., New York, New York). Afterwards, the quantitative real-time PCR(RT-qPCR) primers were synthesized by Xiangyin Biotechnology China (Hangzhou China). RT-qPCR was performed using TB GreenTM Premix Ex TaqTM II (TakaRa Code: DRR820A) following the standard protocol with the 7900HT Fast real-time System (Applied Biosystems, Foster City, CA, USA). The thermal cycling program consisted of 1 cycle at $95^{\circ} \mathrm{C}$ for $1 \mathrm{~min}$, followed by 40 cycles at $95^{\circ} \mathrm{C}$ for $5 \mathrm{~s}$, and then $60^{\circ} \mathrm{C}$ for $30 \mathrm{~s}$. GAPDH was used as the reference gene. The relative gene expression levels were determined according to the critical threshold $(\mathrm{Ct})$ number and calculated using the $2^{-\triangle \Delta \mathrm{Ct}}$ method. The primers for POGZ used for RT-qPCR were as follows: $P O G Z$ F1 ACCCAGTTTGTTAAGCCGACA, $P O G Z$ R1 CTGGAGACTGAACAGCTAGTTG, $P O G Z$ F2 GTGAAGCGACCTGGTGTTACA and POGZ R2 ACATCGTGGACATATTTTCCGTC. The primers for the housekeeping gene were as follows: 5'-TGACTTCAACAGCGACACCCA-3' (GAPDH forward primer) and 5'- CACCCTGTTGCTGTAGCCAAA-3' (GAPDH reverse primer).

\section{Sample collection and high-throughput sequencing}

After the U-2OS and Saos-2 cells were transfected with siPOGZ-2 and siPOGZ-3 and NC was used as a control. Next, RNA was extracted separately for high-throughput sequencing 48 $\mathrm{h}$ later. We used high-throughput sequencing technology to purify mRNA from total RNA and amplified it with PCR technology to build our RNA database. The concentration of the RNA library was detected and diluted to $1 \mathrm{ng} / \mu \mathrm{L}$. After accurate quantification, the insertion size was qualified and used to cluster with the coded samples to form clusters. Finally, these clusters were sequenced to determine the expression of the desired gene expression.

\section{Western blotting}

Cells were lysed with RAPI buffer $48 \mathrm{~h}$ following siRNA transfection, and then centrifuged at $12,000 \mathrm{~g}$ for $10 \mathrm{~min}$, and total protein samples were collected. Protein concentration was determined in each sample using the PierceTM BCA Protein Assay Kit (Thermo Fisher Scientific). Subsequently, $25 \mu \mathrm{g}$ of protein was separated by SDS-PAGE, and then transferred to polyvinylidene difluoride membranes. After being blocked in Tris-buffered saline (TBS) containing 5\% non-fat milk for $1 \mathrm{~h}$ to saturate additional protein binding sites, the blots were incubated with the following primary antibodies: anti-POGZ (1:1,000, Absin, abs132798), antiE-cadherin (1:5,000, Proteintech, cat20874-1-AP), anti-N-cadherin (1:2000, Proteintech, cat22018-1-AP), anti-vimentin (1:2,000, Proteintech, cat10366-AP), anti-cyclinE1 (1:1,000, Proteintech, cat11554-1-AP), and cyclinB1 (1:1,000, Proteintech, cat55004-1-AP). The antibodies were maintained at $4^{\circ} \mathrm{C}$ for $12 \mathrm{~h}$, followed by incubation with horseradish peroxidase-conjugated secondary (anti-mouse or anti-rabbit) IgGs at room temperature for $2 \mathrm{~h}$ or $4 \mathrm{~h}$. The proteins were visualized by using a BM Chemiluminescence Western blotting kit (Roche Diagnostics $\mathrm{GmbH})$. To ensure the equal loading and the accuracy of changes in protein abundance, the level of each protein was normalized to that of GAPDH as a housekeeping control.

\section{Colony formation assay}

Cells were plated on six-well tissue culture plates at a density of 50 cells $/ \mathrm{cm}^{2}$. Fourteen days later, the colonies were fixed with ethanol and stained with $2 \%$ crystal violet, then washed with water to remove the excess dye, and imaged using a scanner. Changes in clonogenicity were quantified by counting the number of colonies, using the Image J software.

\section{Cell proliferation by EdU and Cell Counting Kit-8 (CCK8) assay}

To assess the degree of cell proliferation, $5 \times 10^{3} \mathrm{U}-2 \mathrm{OS}$ and Saos- 2 cells were plated on 24-well plates, and then the cells were incubated under standard conditions in a complete media. After 48 $\mathrm{h}$ following siRNA transfection, cell proliferation was detected based on the incorporation of 5-ethynyl-2'-deoxyuridine (EdU) with the EdU Cell Proliferation Assay Kit. Images were captured using a fluorescence microscope (OLYMPUS, BX53, China). The level of cell proliferation was evaluated using the CCK-8 assay (DOJINDO, Kumamoto, Japan, Cat\# CK04). For this purpose, approximately 3,000 cells in $100 \mu \mathrm{L}$ medium were seeded in each well of a 96-well plate, and three independent parallel experiments were set up. The cells were incubated at $37^{\circ} \mathrm{C}$ in $5 \% \mathrm{CO}_{2}$, and $10 \mu \mathrm{L}$ CCK-8 reagent was added to the wells at $1,2,3,4,5$ and 6 days, and incubated for $2 \mathrm{~h}$. Finally, the absorbance was measured at a wavelength of $450 \mathrm{~nm}$.

\section{Cell migration and invasion assay}

OS cells in $200 \mu \mathrm{L}$ of serum-free DMEM were added to upper chamber of prepared Transwell plates for the migration and invasion assays, and media containing $10 \%$ FBS was added to the lower chamber. The plates were incubated in $5 \% \mathrm{CO} 2$ at $37^{\circ} \mathrm{C}$ overnight. The cells on the upper surface were removed using a cotton bud. The remaining invading cells were fixed and stained with $2 \%$ crystal violet. Five representative fields of view for each membrane were selected, then images were taken via microscopy, and the number of migrating cells was counted using the ImageJ software.

\section{Cell cycle analysis by flow cytometry}

Fixed cells were stained with propidium iodide (PI) (50 $\mu \mathrm{g} / \mathrm{mL}$, Sigma) $48 \mathrm{~h}$ after transfection with siRNA targeting POGZ. The tests were performed in triplicates. To guarantee the accuracy of the cell cycle analysis, the cells that interfered after 48 hours were centrifuged at $1,500 \mathrm{rpm}$ for $5 \mathrm{~min}$ and then resuspended in $500 \mu \mathrm{l}$ of PBS, and $1.5 \mathrm{~mL}$ of a $95 \%$ ethanol solution $\left(-20^{\circ} \mathrm{C}\right.$ pre-cooled) was added to fix the cells at $-20^{\circ} \mathrm{C}$ for $10 \mathrm{~min}$. After centrifugation of the cells centrifuged at $1,500 \mathrm{rpm}$ for $10 \mathrm{~min}$, the supernatant was discarded, and $500 \mu \mathrm{l}$ PBS was added to resuspend the hydrated cells for $10 \mathrm{~min}$. Subsequently, RNase A was added at $37^{\circ} \mathrm{C}$ for $10 \mathrm{~min}$. Ultimately, we added PI $(50 \mu \mathrm{g} / \mathrm{ml})$ to DNA and the content was stained for $15 \mathrm{~min}$. Then, the EdU test was performed. EdU analysis and cell cycle determination were performed using BD CellQuest Pro ${ }^{\mathrm{TM}}$ on a BD FACSCalibur flow cytometer (BD Biosciences, New Jersey, USA) by obtaining at least 20,000 mononuclear cells.

\section{Statistical analyses}

Statistical analyses were performed using the SPSS software version 22.0 and GraphPad Prism 7 software. All the data are expressed as the mean \pm standard deviation. One-way analysis of variance (ANOVA) was conducted to compare the data from multiple groups simultaneously. Student's $t$-test was used for data 
comparison between two groups. For all analyses, two-tailed pvalues below 0.05 were considered statistically significant.

\section{Results}

\section{Identification of genes that are significantly related to} the prognosis of OS

After excluding 244 studies involving cell lines and 31 nonconforming studies in the GEO, we selected 6 studies, whose data were included in the GSE11414, ${ }^{15}$ GSE12865, ${ }^{16}$ GSE14359, ${ }^{17}$ GSE16102, ${ }^{18}$ GSE42352, ${ }^{19,20}$ GSE42572 ${ }^{21}$ datasets, for inclusion in the meta-analysis (Supplementary Figure 1). We selected 10,390 genes from the 6 datasets for meta-analysis and obtained the corresponding negative $\mathrm{Z}$ score. There were no survival data in the six datasets for meta-analysis, so the GSE21257 dataset was used for survival analysis to obtain the $\log 2 \mathrm{HR}$ value corresponding to each gene. Based on the cut-off criteria of a negative $\mathrm{Z}$ score greater than 4.5 and a $\log 2$ hazard ratio (HR) value greater than 1 , we screened out 4 genes that met the criteria (POGZ, CTSE, GALNT14, HSD11B2, Figure 1a). Among these 4 genes, we selected the most significantly upregulated gene (with the most negative $\mathrm{Z}$ score), $P O G Z$ (negative $\mathrm{Z}$ score $=5.06, \log 2 \mathrm{HR}=1.26, \mathrm{p}=0.04$ ). Additionally, the forest plot revealed that within the 6 datasets, $P O G Z$ was steadily upregulated and had no heterogeneity among a
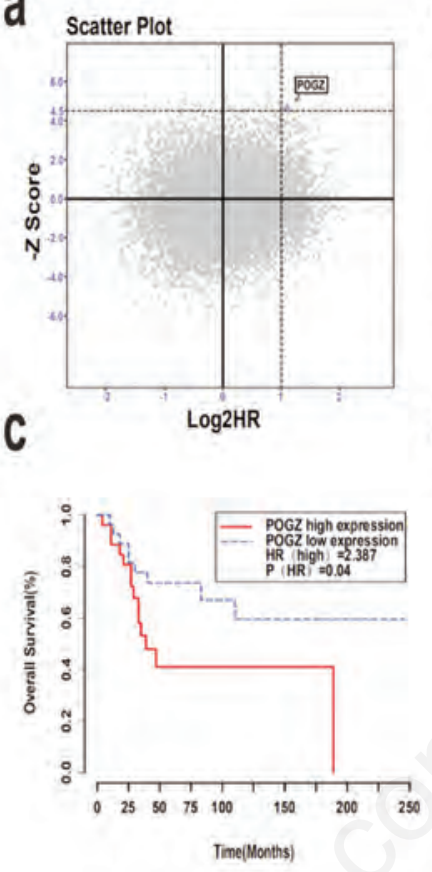

e

b

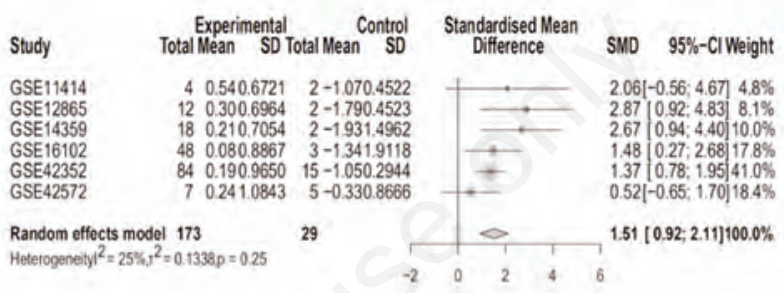

d

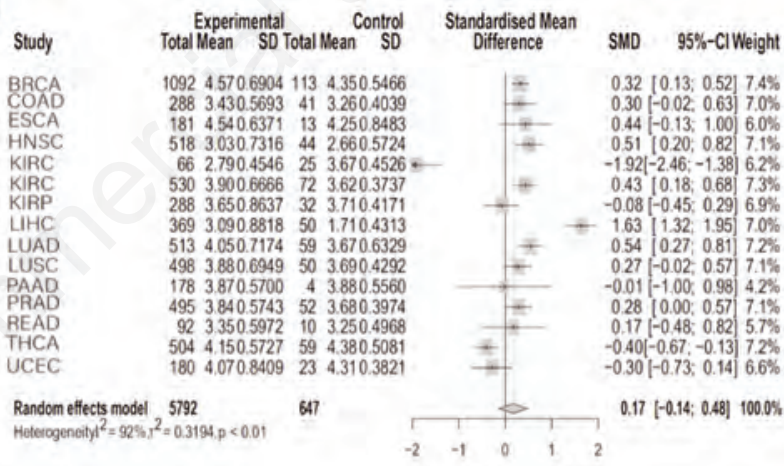

overall survival

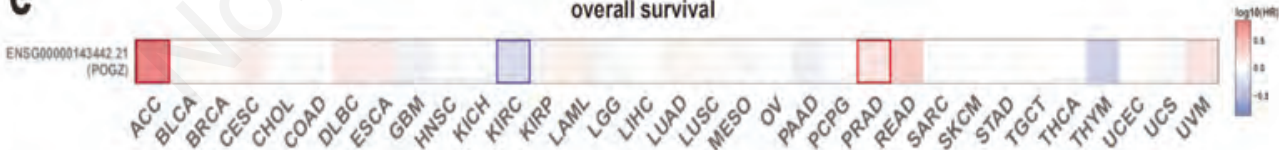

f

disease-free survival

ENSGQ000001434221
(POGZ)

-

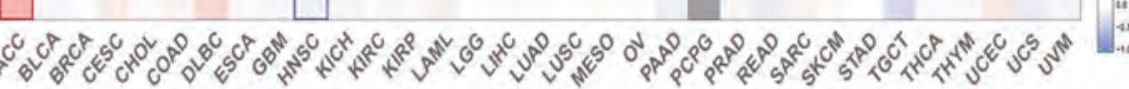

Figure 1. Identify genes that are unfavorable upregulated and associated with a poor prognosis of OS. A) Identifying candidate genes according to the criteria: negative $Z$ score $>4.5$ and $\log 2 \mathrm{HR}>1$; among these 4 genes, POGZ had the most significantly upregulated gene (the most negative $\mathrm{Z}$ score). b) Forest plot of POGZ expression across meta-analysis; the plot revealed that within the 6 datasets, $P O G Z$ was steadily upregulated and had no heterogeneity $\left(I^{2}=25 \%, T 2=0.1338, p=0.25\right)$. c) $\mathrm{KM}$ analysis of overall survival was performed to indicate higher expression of $P O G Z$ were correlated with the poor survival of OS patients in GSE21257. d) Forest plot of $P O G Z$ expression in different types of tumors; BRCA, breast invasive carcinoma; COAD, Colon adenocarcinoma; ESCA, esophageal carcinoma; HNSC, head and neck squamous cell carcinoma; kidney chromophobe-primary tumor; KIRC kidney renal clear cell carcinoma; KIRP, kidney renal papillary cell carcinoma; LIHC, liver hepatocellular carcinoma; LUAD, lung adenocarcinoma; LUSC, lung squamous cell carcinoma; PAAD, pancreatic adenocarcinoma; PRAD, prostate adenocarcinoma; READ, rectum adenocarcinoma; THCA, thyroid carcinoma; UCEC, uterine corpus endometrial carcinoma. e-f) The relationship between high expression of $P O G Z$ and prognosis of multiple tumors was drawn. 
the datasets $\left(I^{2}=25 \%, T^{2}=0.1338, p=0.25\right.$, Figure $\left.1 b\right)$.

As illustrated in Figure 1c, the survival analysis of 53 OS patients from the GSE21257 dataset suggested that the high expression level of $P O G Z$ was significantly related to the poor prognosis of $\mathrm{OS}(\mathrm{p}<0.05, \mathrm{HR}=2.387)$. In addition, we performed the survival analysis using the TARGET OS and GSE39055 datasets. The results also showed a consistent trend (Supplementary Figures 2 and 3). The data of GSE21257, GSE39055 and TARGET OS data were used for meta-analysis. The results showed that $\overline{P O G Z}$ was steadily upregulated without heterogeneity among the datasets $\left(\mathrm{I}^{2}=0 \%, \mathrm{Z}=2.66, \mathrm{p}=0.008\right.$, Supplementary Figure 4).

To explore the effects of $P O G Z$ on a broad range of tumors, we analyzed tumor tissue versus adjacent normal tissue gene expression in 15 tumor types (a total of 7,316 samples), based on the data containing the tumor and controls in The Cancer Genome Atlas (TCGA) database. The results indicated that the expression level of $P O G Z$ in each tumor was unstable (Figure 1d). Compared with that in the control group, $P O G Z$ expression was upregulated in liver cancer $(P<0.05)$ and downregulated in renal chromophobe cell carcinoma $(\mathrm{p}<0.05)$. Moreover, we analyzed the correlation between $P O G Z$ expression levels and multi-tumor overall survival and disease-free survival, and the results confirmed that the upregulation of $P O G Z$ in liver cancer was not related to the prognosis $(\mathrm{p}>0.05$, Figure $1 \mathrm{e}-\mathrm{f})$. Generally, we believe that $P O G Z$ is an unfavorable risk factor for OS.

\section{Verification that $P O G Z$ is upregulated and related to OS metastasis}

In order to verify the upregulation of $P O G Z$ in OS tissue, we performed IHC staining on 41 OS tissues and 19 control tissues. POGZ was overexpressed in OS tissues versus control tissues (Figure 2a). Subsequently, we conducted ROC curve analysis to evaluate the sensitivity and specificity of $P O G Z$ for the diagnosis of OS. The sensitivity and specificity values of the ROC curve were 0.927 and 0.739 , respectively based on the GSE42352 and GSE99671 datasets (Figure 2 b,c).

Moreover, based on 6 datasets including metastatic and non- a

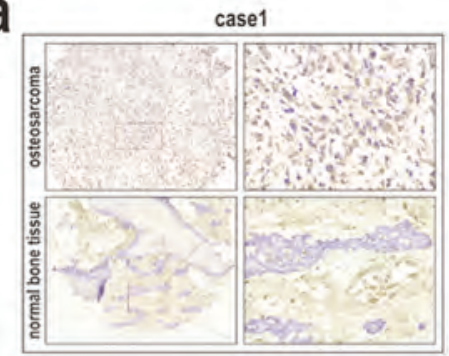

b

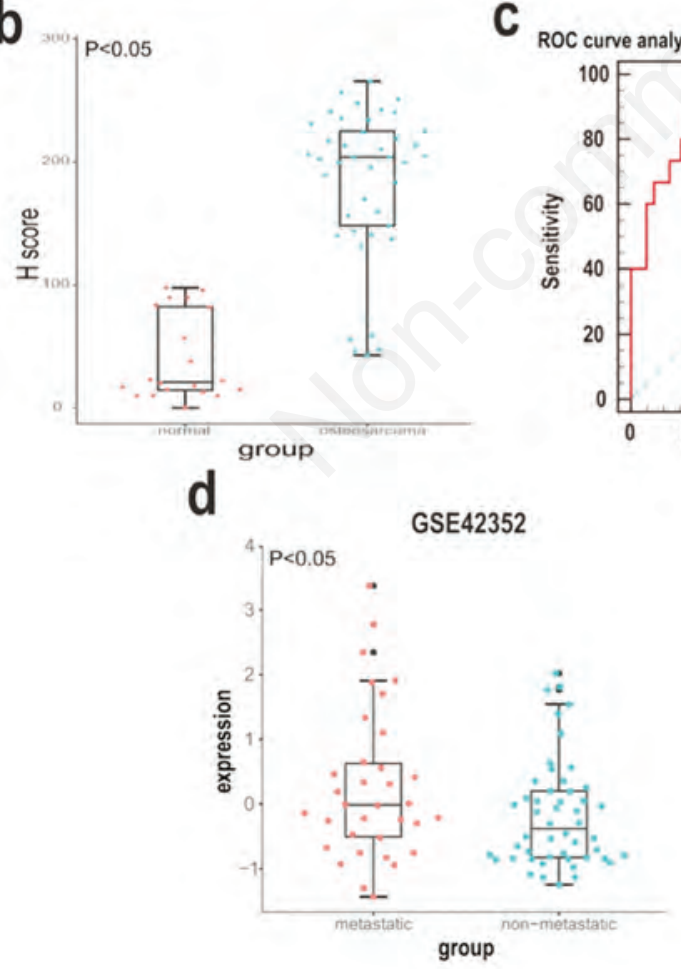

case2

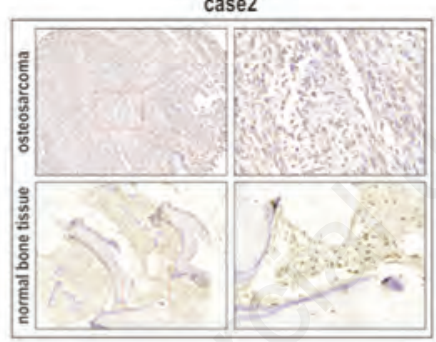

ROC curve analysis in GSE42352:normal vs tumor

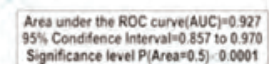

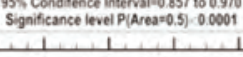
100 -Specificity $(100 \%)$

e

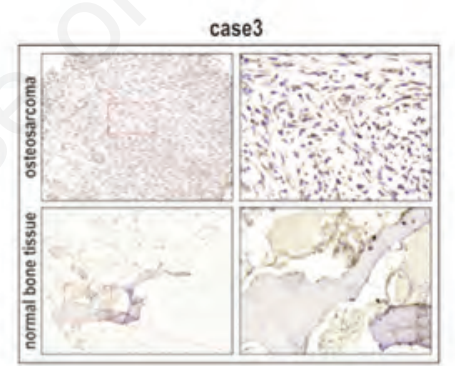

ROC curve analysis in GSE99671:normal vs tumor

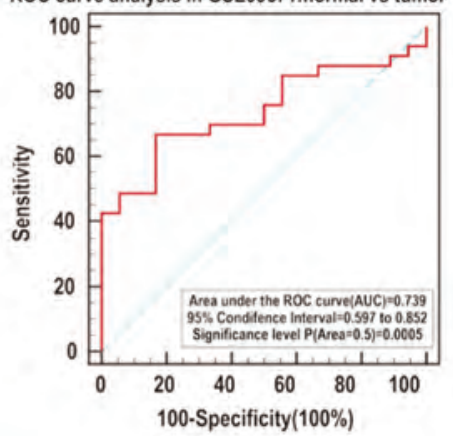

GSE14359

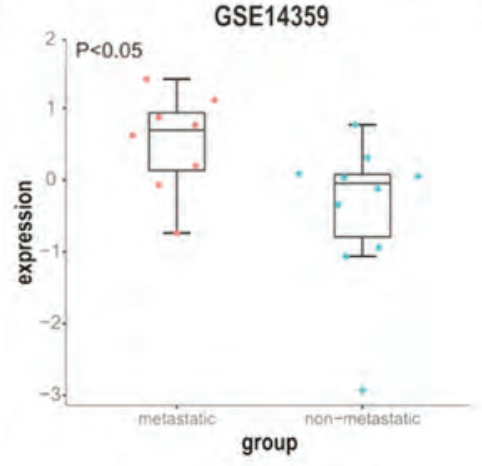

Figure 2. POGZ expression is upregulated in clinical OS samples and related to metastasis. a) Representative images of IHC staining for POGZ in normal bone tissues and OS tissues. b) Quantification of POGZ IHC staining in OS (n=41) and normal tissues (n=19). c,d) The ROC curve from GSE42352 and shows that POGZ is a potential marker enabling to distinguish OS tissues from normal tissues. e,f) High expression of POGZ is associated with metastasis of osteosarcoma; ${ }^{*} \mathrm{p}<0.05,{ }^{* *} \mathrm{p}<0.01,{ }^{* * *} \mathrm{p}<0.001$. 
metastatic OS samples, we developed a forest map of $P O G Z$ expression level analysis (Supplementary Figure 5). After excluding 4 datasets with small sample sizes, we selected the gene expression profiles and corresponding clinical information in the GSE42352 and GSE14359 datasets for the further independent analysis. Next, we utilized $P O G Z$ expression levels to perform a $t$ test and found that $P O G Z$ expression levels were increased in the metastatic groups within the GSE42352 $(\mathrm{t}=1.90 \mathrm{p}=0.031)$ and GSE14359 cohorts $(\mathrm{t}=2.29 \mathrm{p}=0.018)$ were all increased (Figure 2 $\mathrm{d}, \mathrm{e})$. Ultimately, high expression levels of $P O G Z$ play a vital role in the progression of OS.

\section{POGZ knockdown inhibited OS cell proliferation}

To determine the impact of $P O G Z$ on OS proliferation, two siRNA sequences were used to knock down the expression of POGZ in U-2OS and Saos-2 cells. RT-qPCR and Western blotting were carried out to gauge the knockout efficiency (Figure $3 a, b)$. A clonogenic assay proved that compared with that in the NC group, the number of colonies formed by OS cells transfected with siPOGZ-2 and siPOGZ-3 was greatly reduced (Figure $3 \mathrm{c}$ ). The EdU detection method was implemented to evaluate the cell proliferation. This method is an immunochemical detection method that measures the incorporation of nucleotide analogs into newly
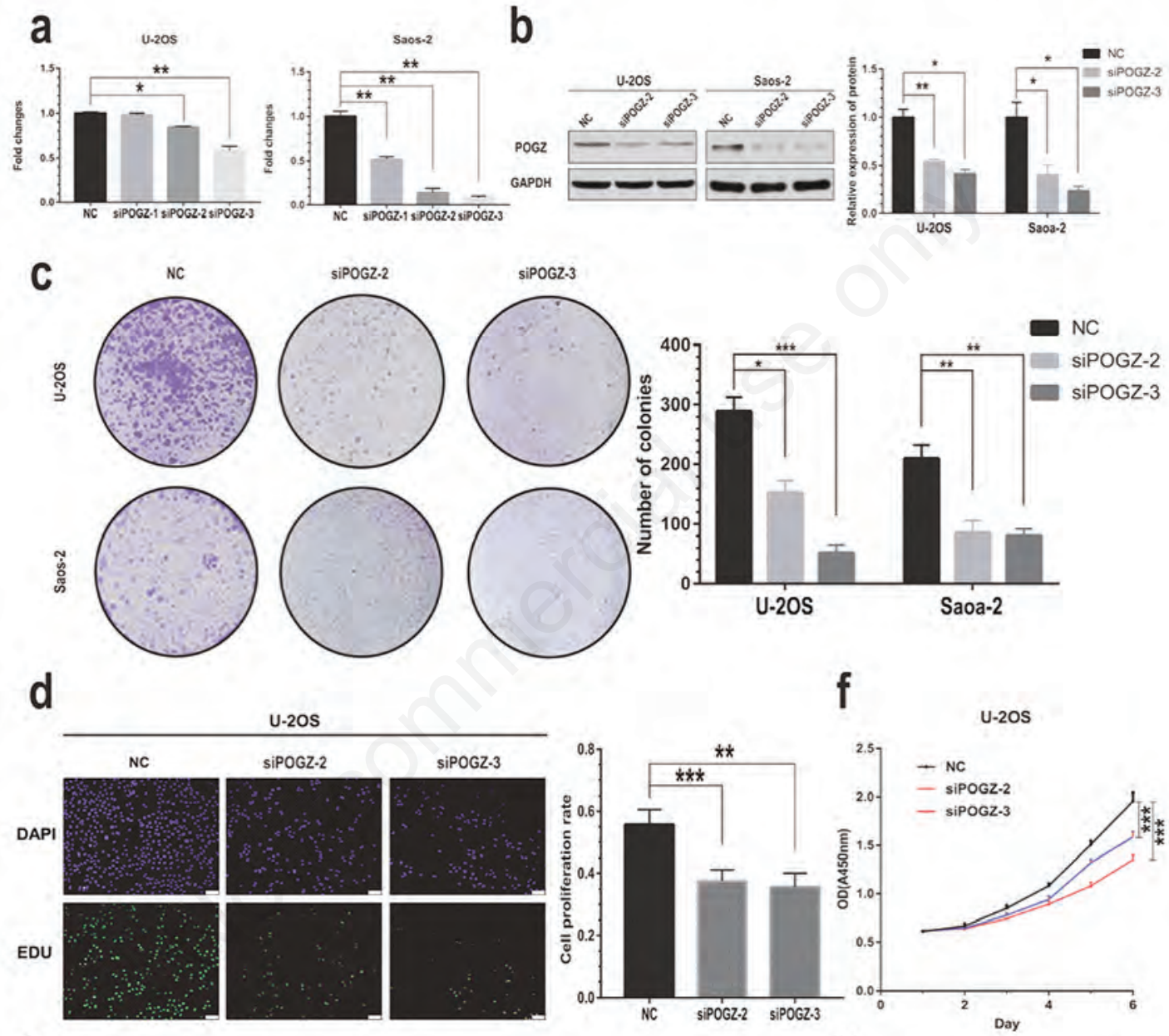

f

e
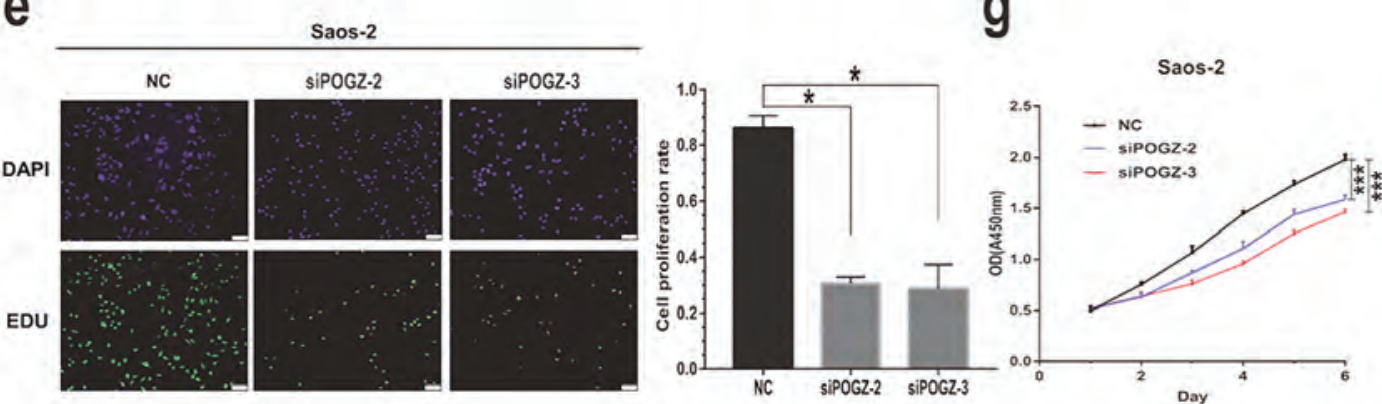

Figure 3. Cell proliferation assay in POGZ inhibited OS cell lines. a) The capability of different siRNAs in terms of downregulating $P O G Z$ expression. b) The protein levels of $P O G Z$ by western blot after different siRNAs knock down. c) Representative clonogenic assay of U-2OS cells and Saos-2 cells expressing the indicated plasmids. Quantitative analysis was performed using the ImageJ software. d,e) EdU assays for proliferation rates. f,g) CCK8 assays for proliferation rates; ${ }^{*} \mathrm{p}<0.05,{ }^{* *} \mathrm{p}<0.01,{ }^{* * *} \mathrm{p}<\mathbf{0 . 0 0 1}$. 
copied DNA. In U-2OS and Saos-2 cells, the percentage of EdUpositive cells treated with siPOGZ-2 and siPOGZ-3 was significantly reduced (Figure $3 \mathrm{~d}, \mathrm{e}$ ). Additionally, we assessed the proliferation of OS cells using the CCK-8 assay. The growth rate of OS cells with $P O G Z$ knockdown was decreased compared to that of control cells (Figure $3 \mathrm{f}, \mathrm{g}$ ). These results indicate that interference with $P O G Z$ expression inhibits OS cell proliferation.

\section{Interference with $P O G Z$ inhibits the migration and invasion of $\mathrm{OS}$ cells}

Migration and invasion are key steps in the development of tumors and metastasis of tumors. In vitro transwell analysis was used to study the effect of $P O G Z$ on the migration and invasion of OS cells. Compared with NC cells, U-2OS and Saos-2 cells in the siPOGZ-2 and siPOGZ-3 groups exhibited remarkably reduced migration and invasion (Figure $4 \mathrm{a}, \mathrm{b}$ ). Furthermore, Western blotting was performed to assess the levels of epithelial-mesenchymal transition (EMT)-related proteins, and the results indicated that vimentin and $\mathrm{N}$-cadherin were both downregulated, while E-cadherin was more upregulated in the U-2OS and Saos-2 cells of the siPOGZ groups than in those of the NC group (Figure 4c). These findings suggest that interference with $P O G Z$ expression inhibits OS cell metastasis.
Genes associated with $P O G Z$ are enriched in cell cycle related processes

Based on the RNA-seq, the KEGG pathway analysis results revealed that the following pathways were positively related to POGZ expression: the DNA replication, homologous recombination, the cell cycle, the Fanconi anemia, RNA transport, spliceosomes pathways. Most of these pathway' terms (cell cycle, DNA replication, homologous recombination, and Fanconi anemia) are related to the cell cycle (Figure $5 \mathrm{a}, \mathrm{b}$ ). To determine which genes in the $P O G Z$ and cell cycle pathways were co-expressed to affect the growth of OS cells, we used the high-throughput sequencing results to perform a differential analysis, which demonstrated that $C C N E 1$ was upregulated and $C C N B 1$ was downregulated (Figure $5 \mathrm{c}$ ). The above results confirm that $P O G Z$ has a regulatory effect on cell cycle pathways and also affects the expression of downstream CCNE1 and CCNB1.

\section{$P O G Z$ controls the cell cycle progression in vitro}

To verify the results of our biological analysis and highthroughput sequencing, we performed a flow cytometric analysis of isolated OS cell cell cycle. OS cells with POGZ knockdown exhibited cell cycle arrest in the $\mathrm{G}_{1}$ phase, confirming the role of $P O G Z$ in regulating cell cycle progression (Figure $5 \mathrm{~d}$ ). Western a

b
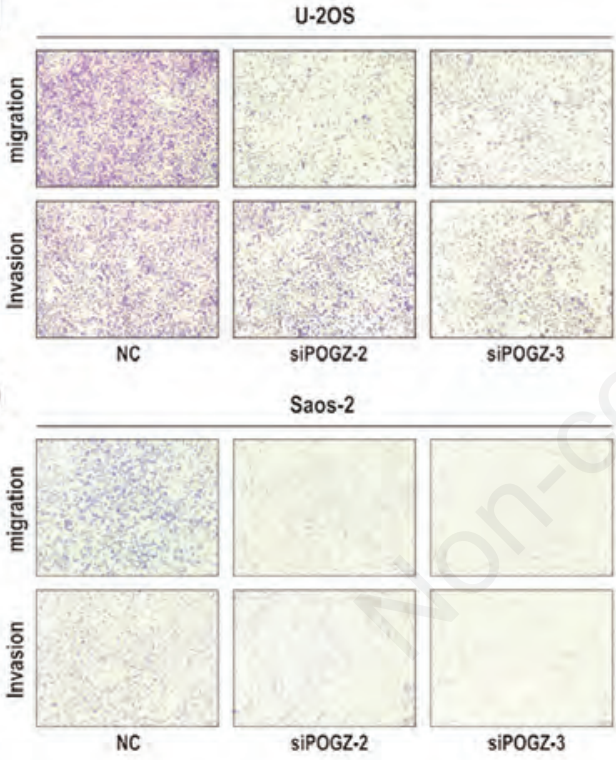

C

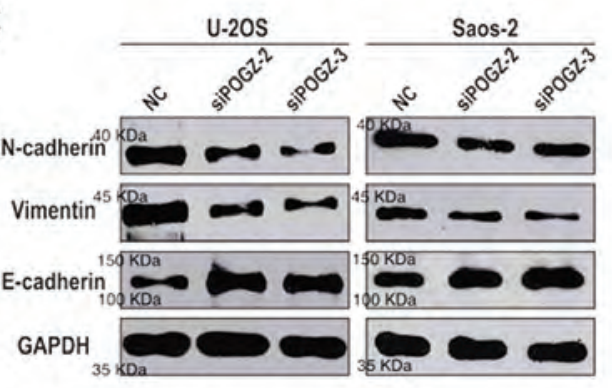

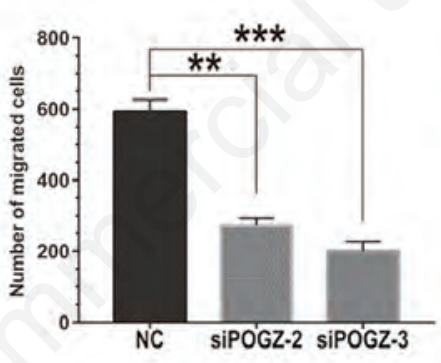
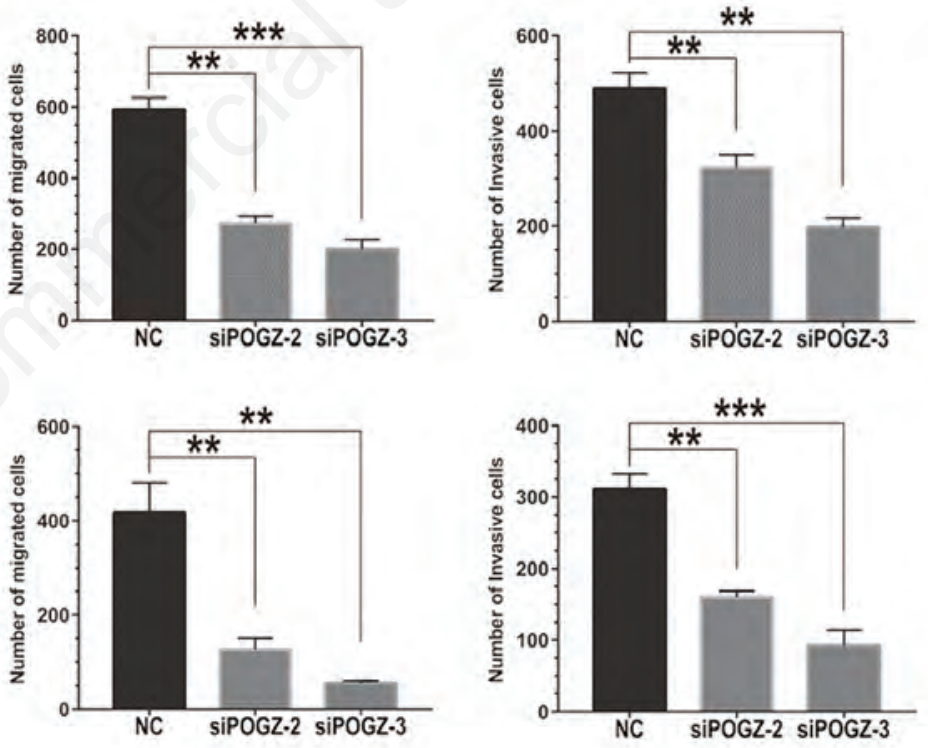

U-20S

Saos-2
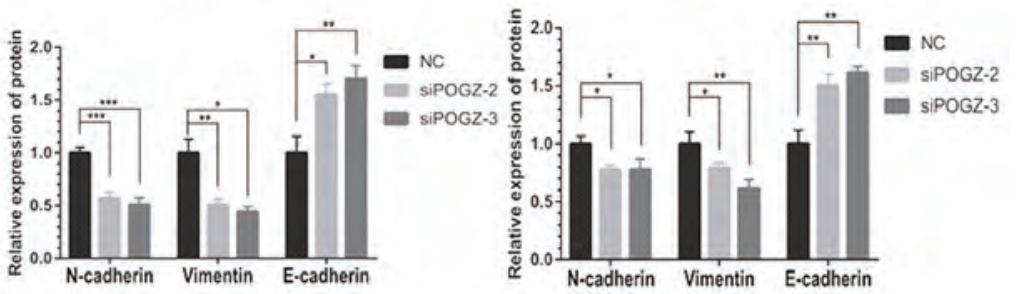

Figure 4. POGZ inhibitions prevent the metastasis and invasion of OS cells. a,b) Transwell assays showed that POGZ inhibitions significantly reduced the migration (upper) and invasion (lower) of OS cells. c,d) EMT markers were detected by Western blot; ${ }^{*}$ p $<0.05$, ${ }^{* *} \mathbf{p}<0.01,{ }^{* * *} \mathbf{p}<0.001$. 
a

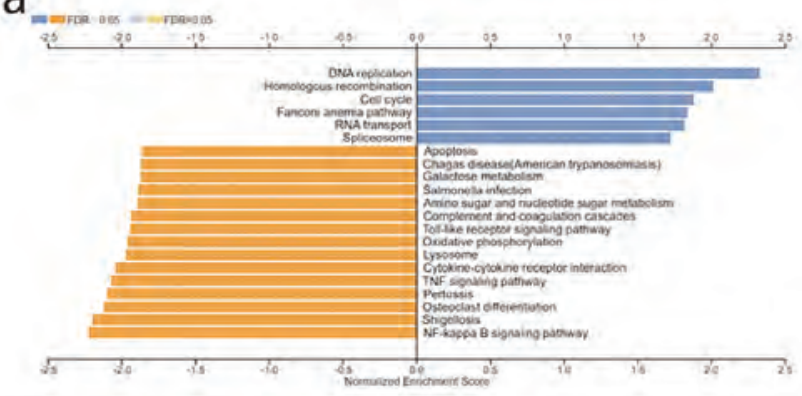

b

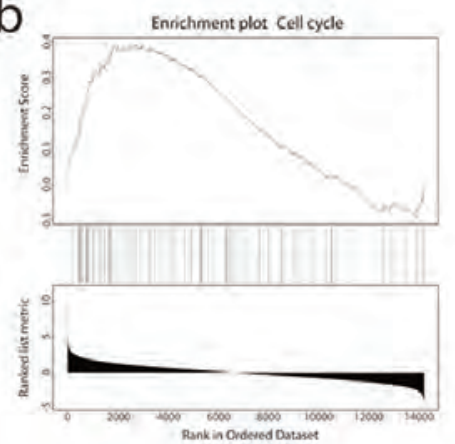

C U.20S

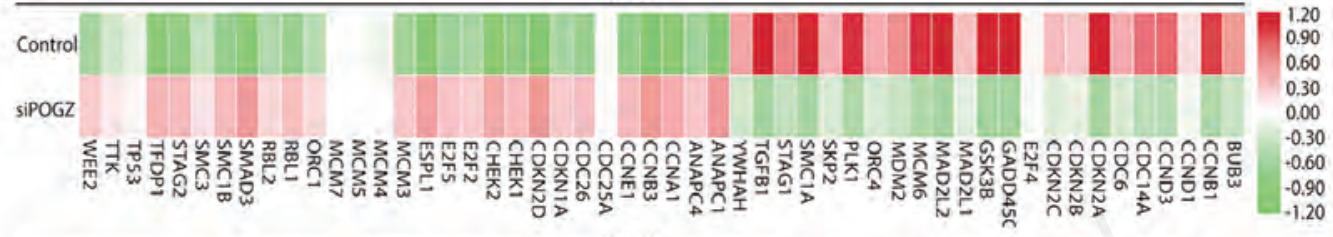

Saos-2

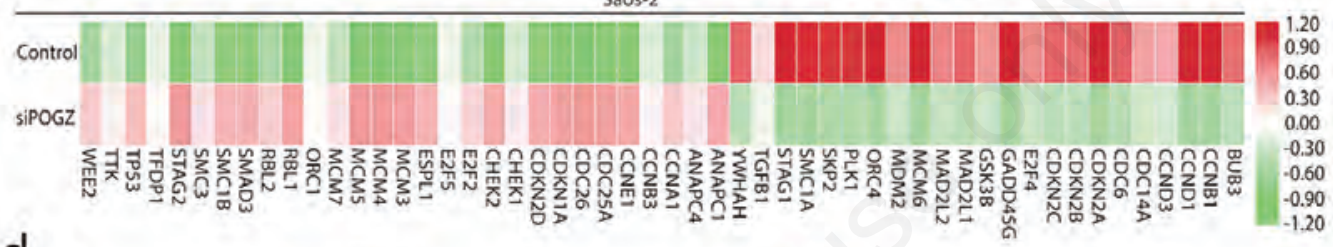

d
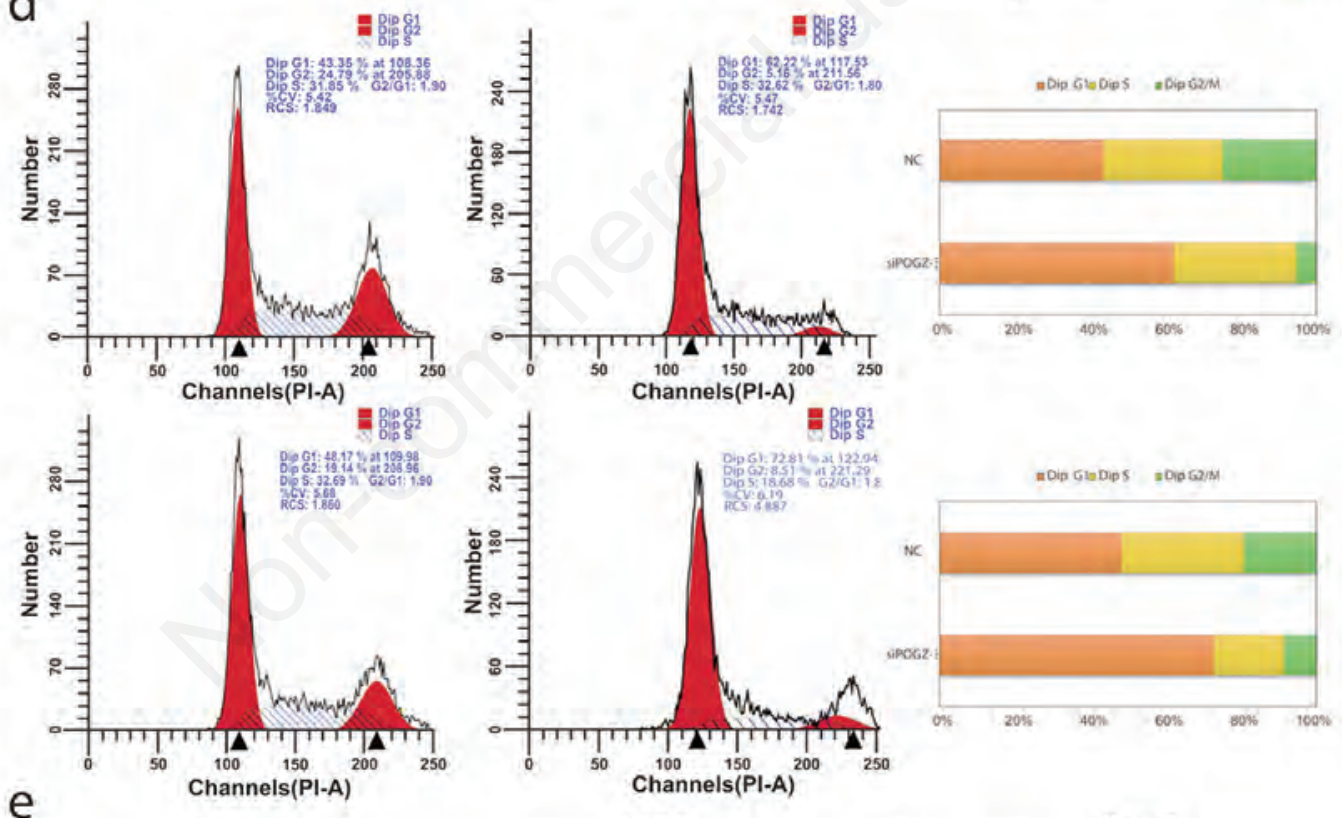

e

U2O-S

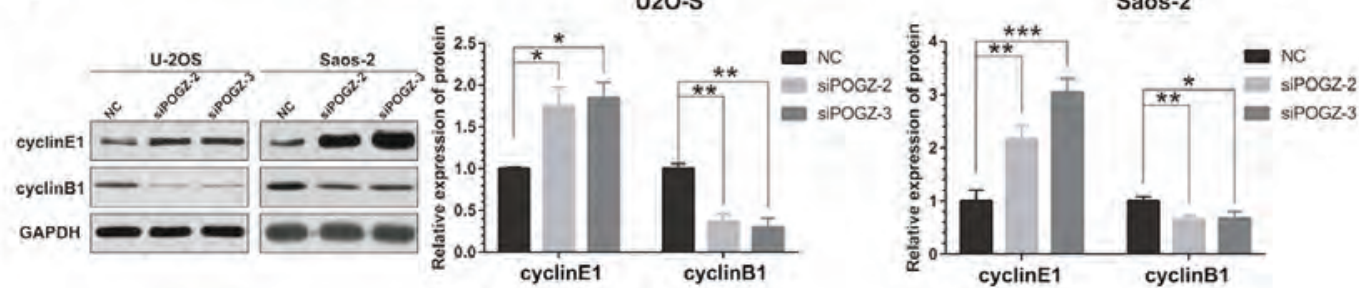

Figure 5. Genes associated with $P O G Z$ were enriched in cell cycle related processes and POGZ controls the cell cycle progression in vitro. a) KEGG pathway enrichment analysis of genes positively and negatively associated with $P O G Z$. b) $P O G Z$ is associated with cell cycle progression. c) Heat map of core-DEGs related to cell cycle process in POGZ inhibited U-2OS and Saos-2 cells; the heat map reveals that $C C N E 1$ was upregulated and $C C N B 1$ was downregulated in OS cells after POGZ inhibition. d) Representative images (left) and quantification (right) of negative control (siPOGZ-3-transfected U-2OS and Saos-2 cells) were analyzed in the cell cycle assay. e) Cell cycle related makers were detected by Western blot; ${ }^{*} \mathrm{p}<0.05,{ }^{* *} \mathrm{p}<0.01,{ }^{* * *} \mathrm{p}<0.001$. 
blot detection of cycle-related proteins revealed that $C C N E 1$ was upregulated and $C C N B 1$ was downregulated in knockdown cells compared to control cells (Figure 5e). Overall, POGZ was shown to affect the cell cycle of OS cells by participating in the regulation of the G1 phase.

\section{Discussion}

$P O G Z$ is an important gene for evaluating the prognosis of OS patients and is highly expressed in OS cells. Inhibiting the expression of $P O G Z$ can reduce OS cell proliferation and tumor metastasis. $P O G Z$ regulates OS cell cycle-related processes, but the mechanism by which OS cells become arrested in the G1 phase after $P O G Z$ inhibition requires more in-depth investigation.

In this study, four candidate genes (POGZ, CTSE, GALNT14, $H S D 11 B 2$ ) met our inclusion criteria: highly expressed in OS and related to a poor prognosis. POGZ contains at least one short region called the zinc finger domain and also contains a centromere protein (CENP)-B-like DNA binding domain and a DDE domain derived from a transposase encoded by a pogo-like DNA transposon. $P O G Z$ is involved in normal kinetochore assembly, cohesion of mitotic sister chromatids, and separation of mitotic chromosomes. ${ }^{26}$ Previous genetic findings have shown that $P O G Z$ is responsible for various neurodevelopmental disorders, including autism spectrum disorders and intellectual disabilities, and is also considered to be related to 'synaptic disorders'. ${ }^{27}$ Since POGZ is the most upregulated of the four target genes, as well as the most harmful to the survival of OS patients, and given that there is no literature report revealing its impact on tumorigenesis, we decided to conduct an in-depth research on it.

Based on the TCGA database, we used Gene Expression Profiling Interactive Analysis (GEPIA) to analyze the relationship between $P O G Z$ expression and the prognosis of multiple tumors. Supplementary Figures 6 and 7 show that $P O G Z$ acts as a survival protective factor in other solid tumors (disease-free survival HR = $0.91, \mathrm{p}=0.017$; overall survival $\mathrm{HR}=0.9, \mathrm{p}=0.0065$ ). Interestingly, these results indicate that high expression of $P O G Z$ is a specific risk factor in OS. Furthermore, based on the gene expression profiles and clinical information of the two datasets, we divided the dataset samples into metastatic and non-metastatic OS groups, and found that the expression of $P O G Z$ was significantly higher in the metastatic OS group.

In terms of molecular phenotype, we established a cell model with POGZ downregulation to explore the role of $P O G Z$ in OS. The proliferation and colony formation of OS cells decreased after POGZ knockdown. Transwell experiments demonstrated that the migration and invasion abilities of OS cells were weakened after POGZ knockdown. EMT is a process involving the invasion and metastasis of various tumors. ${ }^{28}$ In this study, we found that the expression of EMT-related proteins vimentin, N-cadherin and Ecadherin in OS cells was positively correlated with the expression of POGZ. This finding indicates that POGZ could induce the metastasis of OS via EMT.

The function of many oncogenes is to either stimulate cell division or counteract cell cycle arrest. ${ }^{29}$ Western blot analysis of cell cycle-related gene levels by Tanak et al. showed that OS cells that were not treated with a gamma-secretase inhibitor (GSI) had higher expression levels of cyclin E1 and a higher number of cells in the $\mathrm{G}_{1}$ phase than those treated with a GSI treatment. ${ }^{30}$ In an experiment to study the effect of TCTP expression on the growth of OS cells, Shen et al. found that cyclinB1 protein levels in Saos- 2 and U2O-S cells decreased after Lv-shTCTP infection and the proportion of cells in the $\mathrm{G}_{2} / \mathrm{M}$ phase was significantly reduced. ${ }^{31}$ In our study, the KEGG pathway analysis in Figure 5a indicates that the
OS cell cycle pathway is activated, and the first four pathways with the most significant enrichment are all cell cycle related. According to POGZ and OS cell cycle pathway gene co-expression annotation analysis, we found that after knocking down $P O G Z$, CCNE1 was upregulated and $C C N B 1$ was downregulated. Western blotting was used to verify the above results. CCNB1 (cyclin B) is necessary for adequate control of the G2/M transition phase of the cell cycle. ${ }^{32} C C N E 1$ (cyclin E) is a positive regulator of the cell cycle. It promotes the $\mathrm{G}_{1} / \mathrm{S}$ phase transition by binding to $C D K 2$ and activating $C D K 2 .{ }^{33}$ Thus, we believe that $P O G Z$ regulates the OS cells, thereby affecting cell proliferation. However, the limitation of our research was the underlying molecular mechanism was not investigated.

\section{References}

1. Li Y, Nakka M, Kelly AJ, Lau CC, Krailo M, Barkauskas DA, et al. p27 is a candidate prognostic biomarker and metastatic promoter in osteosarcoma. Cancer Res 2016;76:4002-11.

2. Gianferante DM, Mirabello L, Savage SA. Germline and somatic genetics of osteosarcoma - connecting aetiology, biology and therapy. Nat Rev Endocrinol 2017;13:480-91.

3. Rickel K, Fang F, Tao J. Molecular genetics of osteosarcoma. Bone 2017;102:69-79.

4. Tao T, Shen Q, Luo J, Xu Y, Liang W. MicroRNA-125a regulates cell proliferation via directly targeting E2F2 in osteosarcoma. Cell Physiol Biochem 2017;43:768-74.

5. Yoshida A, Fujiwara T, Uotani K, Morita T, Kiyono M, Yokoo $\mathrm{S}$, et al. Clinical and functional significance of intracellular and extracellular microRNA-25-3p in osteosarcoma. Acta Med Okayama 2018;72:165-74.

6. Ma C, Nie XG, Wang YL, Liu XH, Liang X, Zhou QL, et al. CBX3 predicts an unfavorable prognosis and promotes tumorigenesis in osteosarcoma. Mol Med Rep 2019;19:4205-12.

7. Aran V, Devalle S, Meohas W, Heringer M, Cunha Caruso A, Pinheiro Aguiar D, et al. Osteosarcoma, chondrosarcoma and Ewing sarcoma: Clinical aspects, biomarker discovery and liquid biopsy. Crit Rev Oncol Hematol 2021;162:103340.

8. Tian H, Zhou T, Chen H, Li C, Jiang Z, Lao L, et al. Bone morphogenetic protein-2 promotes osteosarcoma growth by promoting epithelial-mesenchymal transition (EMT) through the Wnt/ $\beta$-catenin signaling pathway. J Orthop Res 2019;37:163848.

9. Petryszak R, Burdett T, Fiorelli B, Fonseca NA, GonzalezPorta M, Hastings E, et al. Expression Atlas update--a database of gene and transcript expression from microarray- and sequencing-based functional genomics experiments. Nucleic Acids Res 2014;42(Database issue):D926-32.

10. Han G, Wang Y, Bi W. C-Myc overexpression promotes osteosarcoma cell invasion via activation of MEK-ERK pathway. Oncol. Res 2012;20:149-56.

11. Chen D, Zhao Z, Huang Z, Chen DC, Zhu XX, Wang YZ, et al. Super enhancer inhibitors suppress MYC driven transcriptional amplification and tumor progression in osteosarcoma. Bone Res 2018;6:11.

12. Muff R, Ram Kumar RM, Botter SM, Born W, Fuchs B. Genes regulated in metastatic osteosarcoma: evaluation by microarray analysis in four human and two mouse cell line systems. Sarcoma 2012;2012:937506.

13. Mohamed FEA, Khalil EZI, Toni NDM, Correction to: Caveolin-1 expression together with VEGF can be a predictor for lung metastasis and poor prognosis in osteosarcoma. Pathol Oncol Res 2020;26:2013-4.

14. Isakoff MS, Bielack SS, Meltzer P, Gorlick R. Osteosarcoma: 
Current treatment and a collaborative pathway to success. J Clin Oncol 2015;33:3029-35.

15. Sadikovic B, Yoshimoto M, Al-Romaih K, Maire G, Zielenska M, Squire JA. In vitro analysis of integrated global high-resolution DNA methylation profiling with genomic imbalance and gene expression in osteosarcoma. PLoS One 2008;3:e2834.

16. Sadikovic B, Yoshimoto M, Chilton-MacNeill S, Thorner P, Squire JA, Zielenska M. Identification of interactive networks of gene expression associated with osteosarcoma oncogenesis by integrated molecular profiling. Hum Mol Genet 2009; 18:1962-75.

17. Fritsche-Guenther R, Noske A, Ungethüm U, Kuban RJ, Schlag PM, Tunn PU, et al. De novo expression of EphA2 in osteosarcoma modulates activation of the mitogenic signalling pathway. Histopathology 2010;57:836-50.

18. Paoloni M, Davis S, Lana S, Withrow S, Sangiorgi L, Picci P, et al. Canine tumor cross-species genomics uncovers targets linked to osteosarcoma progression. BMC Genomics 2009;10:625.

19. Kuijjer ML, Peterse EF, van den Akker BE, Briaire-de Bruijn $\mathrm{IH}$, Serra M, Meza-Zepeda LA, et al. IR/IGF1R signaling as potential target for treatment of high-grade osteosarcoma. BMC Cancer 2013;13:245.

20. Kuijjer ML, van den Akker BE, Hilhorst R, Mommersteeg M, Buddingh EP, Serra M, et al. Kinome and mRNA expression profiling of high-grade osteosarcoma cell lines implies Akt signaling as possible target for therapy. BMC Med Genomics 2014;7:4.

21. Buddingh EP, Ruslan SEN, Reijnders CMA, Szuhai K, Kuijjer ML, Roelofs H, et al. Mesenchymal stromal cells of osteosarcoma patients do not show evidence of neoplastic changes during long-term culture. Clin Sarcoma Res 2015;5:16.

22. Buddingh EP, Kuijjer ML, Duim RA, Bürger H, Agelopoulos $\mathrm{K}$, Myklebost $\mathrm{O}$, et al. Tumor-infiltrating macrophages are associated with metastasis suppression in high-grade osteosarcoma: a rationale for treatment with macrophage activating agents. Clin Cancer Res 2011;17:2110-9.
23. Azim HA Jr, Peccatori FA, Brohée S, Branstetter D, Loi S, Viale $\mathrm{G}$, et al. RANK-ligand (RANKL) expression in young breast cancer patients and during pregnancy. Breast Cancer Res 2015;17:24.

24. Ho XD, Phung P, Q Le V, H Nguyen V, Reimann E, Prans E, et al. Whole transcriptome analysis identifies differentially regulated networks between osteosarcoma and normal bone samples. Exp Biol Med (Maywood) 2017;242:1802-11.

25. Ho XD, Nguyen HG, Trinh LH, Reimann E, Prans E, Kõks G, et al. Analysis of the expression of repetitive DNA elements in osteosarcoma. Front Genet 2017;8:193.

26. Nozawa RS, Nagao K, Masuda HT, Iwasaki O, Hirota T, Nozaki N, et al. Human POGZ modulates dissociation of HP1alpha from mitotic chromosome arms through Aurora B activation. Nat Cell Biol 2010;12:719-27.

27. Ibaraki K, Hamada N, Iwamoto I, Ito H, Kawamura N, Morishita R, et al. Expression analyses of POGZ, a responsible gene for neurodevelopmental disorders, during mouse brain development. Dev Neurosci 2019;41:139-48.

28. Pastushenko I, Blanpain C. EMT transition states during tumor progression and metastasis. Trends Cell Biol 2019;29:212-26.

29. Engeland K. Cell cycle arrest through indirect transcriptional repression by p53: I have a DREAM. Cell Death Differ 2018;25:114-32.

30. Tanaka M, Setoguchi T, Hirotsu M, Gao H, Sasaki H, Matsunoshita Y, et al. Inhibition of Notch pathway prevents osteosarcoma growth by cell cycle regulation. Br J Cancer 2009;100:1957-65.

31. Shen JH, Qu CB, Chu HK, Cui MY, Wang YL, Sun YX, et al. siRNA targeting TCTP suppresses osteosarcoma cell growth and induces apoptosis in vitro and in vivo. Biotechnol Appl Biochem 2016;63:5-14.

32. Xie B, Wang S, Jiang N, Li JJ. Cyclin B1/CDK1-regulated mitochondrial bioenergetics in cell cycle progression and tumor resistance. Cancer Lett 2019;443:56-66.

33. Möröy T, Geisen C. Cyclin E. Int J Biochem Cell Biol 2004;36:1424-39.

Received for publication: 17 April 2021. Accepted for publication: 9 July 2021.

This work is licensed under a Creative Commons Attribution-NonCommercial 4.0 International License (CC BY-NC 4.0).

CCopyright: the Author(s), 2021

Licensee PAGEPress, Italy

European Journal of Histochemistry 2021; 65:3264

doi:10.4081/ejh.2021.3264 